\title{
PREDIÇÃO DE GANHOS GENÉTICOS EM PROGÊNIES DE POLINIZAÇÃO ABERTA DE Eucalyptus urograndis CULTIVADAS EM DIFERENTES AMBIENTES E SUBMETIDAS A DIFERENTES PROCEDIMENTOS DE SELEÇÃO ${ }^{1}$
}

\author{
Ricardo Galvão de Freitas ${ }^{2}$, Edmar Soares de Vasconcelos ${ }^{3}$, Cosme Damião Cruz ${ }^{4}$, Antônio Marcos \\ Rosado $^{5}$, Rodrigo Barros Rocha ${ }^{6}$ e Lucas Kenji Takami ${ }^{2}$
}

\begin{abstract}
RESUMO - O objetivo deste estudo foi comparar as estimativas de ganhos genéticos obtidas por diferentes métodos de seleção de genótipos em híbridos de Eucalyptus grandis x Eucalyptus urophylla (E. urograndis). Estimativas de progresso genético foram obtidas considerando-se a prática da seleção massal, seleção massal estratificada, seleção entre e dentro de progênies e seleção combinada, com avaliações em três locais distintos, visando ao desenvolvimento de genótipos com adaptação específica a cada um desses três ambientes. A análise de variância propiciou a constatação da existência de diferenças significativas, a $1 \%$ de probabilidade, pelo teste $\mathrm{F}$, entre os diversos genótipos avaliados nas diferentes localidades. Essa diferença foi verificada nas três variáveis analisadas (diâmetro à altura do peito, altura média das plantas e volume total de madeira), o que permitiu inferir sobre a existência de variabilidade genética entre progênies. A população híbrida estudada possuía bom potencial produtivo e suficiente variabilidade genética a ser explorada por seleção. Os métodos de seleção apresentaram estimativas semelhantes de ganhos genéticos.
\end{abstract}

Palavras-chave: Eucalipto, seleção e ganhos genéticos.

\section{PREDICTION OF GENETIC GAINS IN OPEN POLLINATED PROGENIES OF Eucalyptus urograndis CULTIVATED IN DIFFERENT ENVIRONMENTS AND SUBMITTED TO DIFFERENT SELECTION PROCEDURES}

\begin{abstract}
The objective of this study was to compare the genetic gain estimates obtained by different selection strategies for identification of Eucalyptus grandis $x$ Eucalyptus urophylla genotypes with specific environmental adaptation. The genetic progress obtained by mass selection, stratified mass selection, selection among and within families and combined selection was evaluated in three contrasting environments. Variance analysis showed a significant difference at $1 \%$ of probability according to the F test among the different genotypes evaluated in these three environments. This genetic divergence was observed in all the evaluated traits: diameter at breast height, plant height and wood volume, allowing inferring the existence of genetic variability among families. The hybrid population studied presents good yield potential and genetic variability to be explored by selection. The selection methods showed similar estimates of genetic progress, supporting the decision to use the most practical strategies.
\end{abstract}

Keywords: Eucalyptus, selection and genetic improvement.

\footnotetext{
${ }^{1}$ Recebido em 12.09.2007 e aceito para publicação em 06.03.2009.

${ }^{2}$ Programa de Pós-Graduação em Fitotecnia da Universidade Federal de Viçosa (UFV). E-mail: <ricardogalvaoagro@ yahoo.com.br>.

${ }^{3}$ Departamento de Engenharia Agrícola da Universidade Estadual de Maringá (UEM). E-mail: <vasconceloses@ yahoo.com.br>.

${ }^{4}$ Departamento de Biologia Geral da UFV. E-mail: <cdcruz@ufv.br>.

${ }^{5}$ Doutor, Melhorista da Empresa Celulose-Nipo-Brasileira S.A. (CENIBRA). E-mail: <antonio.rosado@ cenibra.com.br>.

${ }_{5}^{5}$ Pesquisador, Genética e Melhoramento de Plantas EMBRAPA, Rondônia-RO. E-mail: <rodrigo@cpafro.embrapa.br>.
} 


\section{INTRODUÇÃO}

O eucalipto foi introduzido comercialmente no Brasil em 1904, com o objetivo de suprir as necessidades de lenha e postes e das estradas de ferro na Região Sudeste. Na década de 1950, ele passou a ser produzido como matéria-prima para o abastecimento das fábricas de papel e celulose (EMBRAPA, 2006).

As áreas de florestas plantadas no Brasil totalizaram, em 2007, 5.985.396 ha, com o eucalipto ocupando 62,68\%, cerca de 3.751 .867 ha, alcançando, assim, a maior área plantada de eucaliptos do mundo. De 2005 a 2007 , constatou-se incremento na área plantada de 344.128 ha, um acréscimo de $10,1 \%$, sendo Minas Gerais o Estado com maior extensão de áreas plantadas, correspondendo a 28\% em 2007 (Anuário Estatístico da ABRAF 2008 - Ano Base 2007).

O aumento verificado na produção de eucalipto se deve, em grande parte, ao melhoramento genético das diferentes condições ambientais brasileiras. $\mathrm{O}$ melhoramento, associado aos avanços na área de manejo florestal, tem proporcionado o estabelecimento de povoamentos produtivos e adequados a diferentes regiões, condizentes com os usos diversificados da madeira (MARTINS et al., 2005).

Para que seja possível a utilização dos métodos de melhoramento e obtenção de ganhos genéticos, é de fundamental importância verificar a existência de variabilidade dos materiais em estudo. Dessa forma, a obtenção de estimativas dos parâmetros genéticos, como coeficiente de herdabilidade e de variação genética, possui grande importância, sendo necessários na predição dos ganhos e na avaliação da viabilidade do programa de melhoramento, sendo assim úteis para orientar o melhorista na adoção de uma estratégia de melhor eficiência na seleção (VENCOVSKY, 1969).

Em relação às estratégias de seleção, pode-se trabalhar com diferentes métodos, cada um possuindo características e propriedades peculiares. Segundo Allard (1960), a seleção massal é, entre os métodos de melhoramento conhecidos, o mais simples e antigo. Por esse método, plantas são avaliadas e selecionadas fenotipicamente, sendo um método efetivo para caracteres que são facilmente observados ou medidos.

Outro método de seleção é a massal estratificada, que consiste essencialmente em dividir o campo em parcelas ou estratos, sendo a seleção praticada separadamente em cada estrato. Essa estratégia pode ser utilizada no processo de seleção individual. De maneira geral, as populações de melhoramento florestal são normalmente estruturadas em famílias, sendo avaliadas em testes de progênies com informação dentro da parcela e também com informação de procedência, podendo ser de polinização aberta ou controlada. Entre os vários tipos de seleção para estes tipos de população, destacase a seleção combinada, que tem permitido ganhos superiores aos obtidos pela seleção convencional entre e dentro de progênies (PIRES, 1996). A seleção combinada baseia-se no estabelecimento de um índice para cada indivíduo, com base no seu desempenho, considerando os vários fatores experimentais como parcela, bloco e população. Assim, os coeficientes desse índice são estimados a partir dos valores dos próprios indivíduos e também dos valores de seus parentes (MORAES, 1987; PIRES, 1996; CRUZ, 2001).

A seleção direta entre e dentro de progênies é outra alternativa, pois seleciona tanto as melhores progênies quanto os melhores indivíduos dentro delas. Uma das críticas que se faz a esse tipo de seleção é o fato de indivíduos superiores de progênies intermediárias ou indivíduos intermediários de progênies superiores não serem eventualmente considerados na seleção. A seleção combinada é uma alternativa para isso, uma vez que a escolha é feita com base no desempenho individual associado ao desempenho da progênie, em um único estágio. Pela natureza de obtenção, esse tipo de seleção é mais rico em informações e, normalmente, leva a melhores resultados que a seleção entre e dentro (MARTINS et al., 2005).

Este trabalho foi desenvolvido com o objetivo de comparar as estimativas de ganhos genéticos, obtidos por diferentes processos de seleção de genótipos, englobando a seleção massal, a seleção massal estratificada, a seleção entre e dentro de progênies e a seleção combinada em progênies de Eucalyptus urograndis, avaliadas em três locais distintos.

\section{MATERIAL E MÉTODOS}

Os testes de progênies de polinização aberta do híbrido Eucalyptus grandis x E. urophylla (E. urograndis) foram instalados em três locais de experimentação pela Celulose Nipo-Brasileira S.A. (CENIBRA). O espaçamento utilizado foi $3 \mathrm{~m} \mathrm{x} 2 \mathrm{~m}$. Locais de experimentação: Local 1 - referente ao Projeto Milagre, localizado na região de Belo Oriente, MG, 
Latitute: -19 13' 12', Longitude: 42²9' 01', posição de baixada, plantado em novembro de 2002 numa área de 3,6 ha; Local 2 - referente ao Projeto Inhambu, localizado na região de Guanhães, MG, Latitute: -18 46' 30', Longitude: $42^{\circ} 55^{\prime} 57^{\prime}$, posição de encosta, plantado em novembro de 2002 numa área de 5,47 ha; e Local 3 - referente ao Projeto Ribeirão do Boi, localizado na região de Ipaba-MG, Latitute: $-19^{\circ} 24^{\prime} 49^{\prime \prime}$, Longitude: $42^{\circ} 25^{\prime} 10^{\prime \prime}$, posição de encosta, plantado em dezembro de 2002, numa área de 4,82 ha.

Os ensaios foram conduzidos no delineamento de blocos ao acaso, obtendo-se dados dendrométricos individuais de diâmetro à altura do peito (DAP, em $\mathrm{cm}$ ), altura das plantas (ALT, em $\mathrm{m}$ ) e volume (VOL, $\mathrm{em} \mathrm{m}^{3}$ ) em todas as cinco plantas de cada parcela. Cada ensaio foi constituído de número diferente de progênies (Belo Oriente-77, Guanhães - 177 e Ipaba - 186), avaliadas em parcelas de cinco plantas com 36 meses de idade.

\subsection{Análises Estatísticas}

Os dados obtidos nas três características avaliadas (DAP,ALTe VOL) foram submetidos à análise de variância, conforme o seguinte modelo (CRUZ et al., 2004):

$$
Y_{i j k}=m+G_{i}+B_{j}+\varepsilon_{i j}+\delta_{i j k}
$$

em que:

$\mathrm{Y}_{\mathrm{ijk}}=$ observação referente ao indivíduo $\mathrm{k}$, da família i na repetição j;

M= média geral do experimento;

$\mathrm{G}_{\mathrm{i}}=$ efeito do i-ésima progênie considerado aleatório,
$\mathrm{G}_{\mathrm{i}} \sim \operatorname{NID}\left(0, \sigma_{g}^{2}\right)$

$\mathrm{B}_{\mathrm{j}}=$ efeito do j-ésimo bloco, considerado aleatório, $\mathrm{B}_{\mathrm{j}} \sim \operatorname{NID}\left(0, \sigma_{b}^{2}\right)$

$\varepsilon_{\mathrm{ij}}=$ efeito aleatório da variação entre parcelas, $\mathrm{G}_{\mathrm{i}} \sim \mathrm{NID}\left(0, \sigma_{e}^{2}\right) ; \mathrm{e}$

$\delta_{\mathrm{ijk}}=$ efeito aleatório da variação entre plantas dentro da parcela, $\mathrm{G}_{\mathrm{i}} \sim \operatorname{NID}\left(0, \sigma_{d}^{2}\right)$.

A análise de variância das características DAP, ALT e VOL seguiu o esquema apresentado na Tabela 1. Nessa Tabela também se encontram as esperanças dos quadrados médios.

Foram estimados os seguintes parâmetros: variância genética entre progênies $\left(\sigma_{e}^{2}\right)$; variância genética dentro de progênies $\left(\sigma_{d}^{2}\right)$; variância fenotípica dentro de progênies $\left(\sigma_{f d}^{2}\right)$; variância residual $\left(\sigma_{r}^{2}\right)$; variância total $\left(\sigma_{t o t}^{2}\right)$; herdabilidade considerando como unidade de seleção a média da progênie $\left(\sigma_{m f}^{2}\right)$; herdabilidade na unidade de seleção dos valores dentro da progênie $\left(\sigma_{d f}^{2}\right)$; herdabilidade em que a unidade de seleção foi o indivíduo no bloco $\left(\sigma_{I b}^{2}\right)$; herdabilidade em que a unidade de seleção foi o indivíduo no experimento como um todo $\left(\sigma_{\text {Iexp }}^{2}\right)$; coeficiente de variação experimental $(\mathrm{CVe})$; coeficiente de variação genético entre progênies $(\mathrm{CVg})$; coeficiente de variação genético dentro de progênies $(\mathrm{CVd})$; relação entre $\mathrm{CVg}$ e CVe; e também a relação entre CVd e CVe. Os parâmetros foram estimados conforme apresentado na Tabela 2.

Tabela 1 - Esquema da análise de variância realizada com os dados das variáveis DAP, ALT e VOL, obtidos das progênies de meios-irmãos de eucalipto conduzidas em Belo Oriente, Guanhães e Ipaba

Table 1 - Variance analysis scheme using data of the variables DBH (Diameter at Breast Height), H (Plant Height) and VOL (Wood Volume), obtained from half-sib Eucalyptus progenies planted in Belo Oriente, Guanhães and Ipaba

\begin{tabular}{|c|c|c|c|c|}
\hline Fonte de Variação & GL & QM & $\mathrm{E}(\mathrm{QM})$ & $\mathrm{F}$ \\
\hline Source of Variation & $D F$ & $M S$ & $E(M S)$ & $F$ \\
\hline $\begin{array}{l}\text { Blocos } \\
\text { Blocks }\end{array}$ & $r-1$ & $\begin{array}{l}\text { QMB } \\
M S B\end{array}$ & $\sigma_{d}^{2}+\mathrm{n} \sigma_{e}^{2}+\mathrm{ng} \sigma_{b}^{2}$ & \\
\hline $\begin{array}{l}\text { Genótipos } \\
\text { Genotypes }\end{array}$ & $g-1$ & $\begin{array}{l}\text { QMG } \\
M S G\end{array}$ & $\sigma_{d}^{2}+\mathrm{n} \sigma_{e}^{2}+\mathrm{nr} \sigma_{g}^{2}$ & $\begin{array}{l}\text { QMG/QME } \\
M S G / M S E\end{array}$ \\
\hline $\begin{array}{l}\text { Entre Parcelas } \\
\text { Among Plots }\end{array}$ & $(\mathrm{r}-1)(\mathrm{g}-1)$ & $\begin{array}{l}\text { QME } \\
M S E\end{array}$ & $\sigma_{d}^{2}+\mathrm{n}_{\sigma_{e}^{2}}^{2}$ & \\
\hline $\begin{array}{l}\text { Dentro de Parcelas } \\
\text { Within Plots }\end{array}$ & $(n-1) r g$ & $\begin{array}{l}\text { QMD } \\
M S D\end{array}$ & $\sigma_{d}^{2}$ & \\
\hline
\end{tabular}

Total

$\operatorname{rgn}-1$

Total

QM: quadrado médio; $\mathrm{E}(\mathrm{QM})$ : esperança do quadrado médio; $\sigma_{d}^{2}:$ variância devido a variação dentro de parcelas; $\sigma_{e}^{2}:$ variância devido ao erro entre parcelas; $\sigma_{b}^{2}$ : variância devido ao efeito de bloco; e $\sigma_{g}^{2}$ : variância genética; r: número de blocos; g: número de genótipos; e n: número de plantas por parcela.

MS: mean square; E(MS): mean square expectation; $\sigma_{d}^{2}:$ variance within plots; $\sigma_{e}^{2}:$ variance due to error among plots; $\sigma_{b}^{2}:$ variance due to block effect; and $\sigma_{g}^{2}$ : genetic variance. $r$ : number of blocks; g: number of genotypes; and n: number of plants per plot. 
Tabela 2 - Estimadores da variância genética entre progênies, variâncias fenotípicas dentro de progênies $\left(\sigma_{d}^{2}\right)$, residual $\left(\sigma_{e}^{2}\right)$, total $\left(\sigma_{t o t}^{2}\right)$ e de blocos $\left(\sigma_{b}^{2}\right)$; estimativa da herdabilidade, sendo a unidade de seleção média entre progênies $\left(\sigma_{m f}^{2}\right)$, dentro de progênies $\left(\sigma_{d f}^{2}\right)$, indivíduo no bloco $\left(\sigma_{l b}^{2}\right)$, indivíduo no experimento $\left(\sigma_{\text {Iexp }}^{2}\right)$; coeficiente experimental $(\mathrm{CVe})$, coeficiente de variação genético entre progênies $\left(\mathrm{CV}_{\mathrm{GE}}\right) \mathrm{e}$ coeficiente de variação genético dentro de progênies $\left(\mathrm{CV}_{\mathrm{GD}}\right)$

Table 2 -Estimators of the genetic variance among progenies, phenotypic variances within progenies $\left(\sigma_{d}^{2}\right)$, residual $\left(\sigma_{e}^{2}\right)$, total $\left(\sigma_{\text {tot }}^{2}\right)$ and block $\left(\sigma_{b}^{2}\right)$; estimate of heritability as the unit of average selection among progenies $\left(\sigma_{m f}^{2}\right)$, within progenies $\left(\sigma_{d f}^{2}\right)$, individual in the block $\left(\sigma_{I b}^{2}\right)$, individual in the experiment $\left(\sigma_{\text {Iexp }}^{2}\right)$; experimental coefficient (CVe), genetic variation coefficient among progenies $\left(C V_{G E}\right)$ and genetic variation coefficient within progenies $\left(C V_{G D}\right)$.

\begin{tabular}{|c|c|}
\hline $\begin{array}{l}\text { Parâmetro Genético } \\
\text { Genetic Parameter }\end{array}$ & $\begin{array}{l}\text { Estimador do Parâmetro Genético } \\
\text { Estimator of the Genetic Parameter }\end{array}$ \\
\hline$\sigma_{g}^{2}$ & $\begin{array}{l}(\mathrm{QMG}-\mathrm{QME}) / \mathrm{nr} \\
(\mathrm{MSG}-\mathrm{MSE}) / \mathrm{nr}\end{array}$ \\
\hline$\sigma_{b}^{2}$ & $\begin{array}{l}\text { (QMB-QME)/ng } \\
(\mathrm{MSB}-\mathrm{MSE}) / \mathrm{ng}\end{array}$ \\
\hline$\sigma_{d}^{2}$ & $\begin{array}{l}\text { QMD } \\
\text { MSD }\end{array}$ \\
\hline$\sigma_{\mathrm{e}}^{2}$ & $\begin{array}{c}(\mathrm{QME}-\mathrm{QMD}) / \mathrm{n} \\
(\mathrm{MSE}-\mathrm{MSD}) / \mathrm{n}\end{array}$ \\
\hline$\sigma_{t o t}^{2}$ & $\hat{\sigma}_{b}^{2}+\hat{\sigma}_{d}^{2}+\hat{\sigma}_{d}^{2}+\hat{\sigma}_{g}^{2}$ \\
\hline$h^{2}$ & $\hat{\sigma}_{\mathrm{g}}^{2} /(\mathrm{QMG} / \mathrm{nr})$ \\
\hline$n_{m f}$ & $\hat{\sigma}_{\mathrm{g}}^{2} /(\mathrm{MSG} / \mathrm{nr})$ \\
\hline$h_{d f}^{2}$ & $3 \hat{\sigma}_{g}^{2} /\left(\hat{\sigma}_{d}^{2}\right)$ \\
\hline$h_{I b}^{2}$ & $4 \hat{\sigma}_{g}^{2} /\left(\hat{\sigma}_{g}^{2}+\hat{\sigma}_{d}^{2}+\hat{\sigma}_{e}^{2}\right)$ \\
\hline$h_{I \text { exp }}^{2}$ & $4 \hat{\sigma}_{g}^{2} / \hat{\sigma}_{\text {tot }}^{2}$ \\
\hline $\mathrm{CV}$ & $\begin{array}{c}(100 \sqrt{ }(\mathrm{QME} / \mathrm{n})) / \text { média } \\
(100 \sqrt{ }(\mathrm{MSE} / \mathrm{n})) / \text { mean } \\
\left(100 \sqrt{ } \hat{\sigma}_{\mathrm{g}}^{2}\right) / \mathrm{média}\end{array}$ \\
\hline$C V_{\mathrm{GE}}$ & $\left(100 \sqrt{ } \hat{\sigma}_{g}^{2}\right) /$ mean \\
\hline & $\left(100 \sqrt{ }{\sigma_{g d}^{2}}_{g d}\right) /$ média \\
\hline$C V_{\mathrm{GD}}$ & $\left(100 \sqrt{ } \bar{\sigma}_{g d}^{2}\right) /$ mean \\
\hline
\end{tabular}

\subsection{Progresso com a seleção}

Todas as características foram selecionadas de modo a se obter acréscimo em suas médias originais. Fez-se uso de diferentes metodologias de seleção, obtendo diferentes ganhos proporcionados por cada uma delas. As metodologias de seleção encontramse descritas a seguir.

\subsubsection{Seleção massal}

Na seleção massal, os indivíduos foram selecionados de acordo com o valor fenotípico apresentado em cada característica, independentemente da progênie a que pertenciam. Efetuou-se a seleção dos $25 \%$ indivíduos de melhor resposta, em relação a cada uma das características avaliadas.

As estimativas dos ganhos com essa seleção foram estimadas para cada variável, por meio das seguintes expressões (CRUZ et al., 2004):

$$
\mathrm{GS}_{\mathrm{m}}(\%)=\frac{100 \mathrm{GS}_{\mathrm{m}}}{\overline{\mathrm{X}}_{0}} \text { sendo, } \mathrm{GS}_{\mathrm{m}}=\mathrm{h}_{\operatorname{lexp}}^{2} \mathrm{DS}
$$

em que:

$\mathrm{GS}_{\mathrm{m}=}$ ganho com a seleção massal; DS $=\left(\overline{\mathrm{X}}_{\mathrm{S}}-\overline{\mathrm{X}}_{\mathrm{o}}\right)$ : diferencial de seleção;

$\mathrm{h}_{\text {Iexp= }}^{2}$ herdabilidade em que a unidade de seleção foi o indivíduo no experimento como um todo;

$\bar{X}_{\mathrm{S}}, \overline{\mathrm{X}}_{\mathrm{o}}=$ média dos indivíduos selecionados e média original dos indivíduos avaliados, respectivamente.

\subsubsection{Seleção massal estratificada}

Na seleção massal estratificada, os genótipos foram ordenados dentro de cada bloco, independentemente da progênie. A seguir, realizou-se a seleção do indivíduo de melhor fenótipo, obtendo ao final oito plantas selecionadas. Após selecionar esses indivíduos, voltouse ao bloco 1 e realizou-se nova seleção, assim como nos demais blocos, formando-se o segundo grupo dos oito melhores fenótipos. Esse procedimento foi repetido até selecionar-se $25 \%$ do número total de plantas avaliadas. As estimativas dos ganhos com a seleção massal estratificada foram obtidas conforme se segue:

$$
\mathrm{GS}_{\mathrm{me}}(\%)=\frac{100 \mathrm{GS}}{\overline{\mathrm{me}}} \overline{\mathrm{X}_{0}} \text { sendo } \mathrm{GS}_{\mathrm{me}}=\mathrm{h}_{1 \mathrm{~b}}^{2} \mathrm{DS}
$$

em que:

$\mathrm{GS}_{\mathrm{me}}$ : ganho com a seleção massal estratificada; e

$\mathrm{h}^{2}{ }_{\mathrm{Ib}}$ herdabilidade em que a unidade de seleção foi o indivíduo no bloco. 


\subsubsection{Seleção entre e dentro de progênies}

Nesta metodologia, realizou-se inicialmente a seleção das progênies superiores, restando $25 \%$ do total de progênies. Posteriormente, praticou-se seleção dentro dessas progênies até alcançar um total de $25 \%$ de todos os genótipos avaliados. As estimativas dos ganhos com a seleção entre e dentro das progênies foram obtidas de acordo com as seguintes expressões (CRUZ et al., 2004):

Seleção entre:

$$
\mathrm{GS}_{\mathrm{e}}(\%)=\frac{100 \mathrm{GS}_{\mathrm{e}}}{\overline{\mathrm{X}}_{0}} \text { sendo } \mathrm{GS}_{\mathrm{e}}=\mathrm{h}_{\mathrm{mf}}^{2} \mathrm{DS}
$$

Seleção dentro:

$$
\mathrm{GS}_{\mathrm{d}}(\%)=\frac{100 \mathrm{GS}_{\mathrm{d}}}{\overline{\mathrm{X}}_{0}} \text { sendo } \mathrm{GS}_{\mathrm{d}}=\mathrm{h}_{\mathrm{df}}^{2} \mathrm{DS}_{\mathrm{m}}
$$

Seleção entre e dentro:

$$
\mathrm{GS}_{\mathrm{ed}}=\mathrm{GS}_{\mathrm{e}}+\mathrm{GS}_{\mathrm{d}}
$$

em que:

$\mathrm{GS}_{\mathrm{e}}$ : ganho de seleção entre progênies;

$\mathrm{GS}_{\mathrm{d}}$ : ganho de seleção dentro de progênies; e

$\mathrm{GS}_{\mathrm{ed}}$ : ganho de seleção entre e dentro de progênies.

\subsubsection{Seleção combinada}

Realizou-se a seleção combinada, a qual consiste no estabelecimento de um índice para cada indivíduo, para cada característica. Os pesos que compõem esse índice foram obtidos do próprio indivíduo, sendo adotado o índice proposto por Pires et al.(1996), dado por:

$$
I_{i j k}=b_{i}\left(Y_{i j k}-\bar{Y}_{. j .}\right)+b_{f}\left(\bar{Y}_{i . .}-\bar{Y}_{\ldots}\right)
$$

em que:

$\mathrm{I}_{\mathrm{ijk}}=$ índice estimador do valor genético da k-ésima planta, na j-ésima repetição da i-ésima progênie;

$\mathrm{Y}_{\mathrm{ijk}}=$ valor fenotípico do indivíduo ijk;

$\overline{\mathrm{Y}}_{\mathrm{j}}=$ média da repetição $\mathrm{j}$ a que pertence o indivíduo ijk;

$\overline{\mathrm{Y}}_{\mathrm{i} . .}=$ média da progênie $\mathrm{i}$;

$\bar{Y}_{\ldots}=$ média geral do experimento; $\mathrm{e}$

$\mathrm{b}_{\mathrm{i}}$ e $\mathrm{b}_{\mathrm{f}}$ : pesos atribuídos à seleção de indivíduos.

Obteve-se o índice de seleção combinado que possibilitou a melhor predição do valor genotípico individual. Para isso, fez-se uso da teoria de índice de seleção, de maneira que o ganho por seleção foi obtido por:

$$
\mathrm{GS}_{\mathrm{IC}}=\frac{\mathrm{Côv}_{\mathrm{g}}\left(\mathrm{IC}_{\mathrm{ijk}}, \mathrm{g}_{\mathrm{ijk}}\right)}{\hat{\mathrm{V}}\left(\mathrm{IC}_{\mathrm{ijk}}\right)} \mathrm{DS}_{\mathrm{IC}}
$$

em que:

Côv ${ }_{g}\left(I C_{i j k}, g_{i j k}\right)=$ covariância genética aditiva entre os escores do índice de seleção combinada e os respectivos valores genéticos dos indivíduos;

$\hat{\mathrm{V}}\left(\mid \mathrm{C}_{\mathrm{ijk}}\right)=$ variância dos valores do índice de seleção; e

$\mathrm{DS}_{\mathrm{IC}}=$ diferencial de seleção, obtido a partir dos escores do índice combinado.

As análises estatísticas foram realizadas com o auxílio do aplicativo computacional GENES (CRUZ, 2001).

\section{RESULTADOS E DISCUSSÃO}

Na análise de variância, constatou-se a existência de diferenças significativas no nível de $1 \%$ de probabilidade pelo teste $\mathrm{F}$, nos materiais avaliados nos diferentes locais (Tabela 3). Essa diferença foi verificada em todas as variáveis consideradas (DAP, ALT e VOL) permitindo inferir acerca da existência de diferença genética entre as progênies. A existência de variância é uma premissa básica para o melhoramento genético e subsidia o progresso genético com a prática da seleção (CRUZ et al., 2004).

Estudo realizado por Rocha, M.G.B. et al. (2007) teve como objetivo selecionar genitores de E. urophylla e de $E$. grandis de maior divergência genética, visando à produção de híbridos interespecíficos. As avaliações foram feitas aos 58 meses de idade e as médias da característica DAP apresentaram em E. urophylla e $E$. grandis 11,90 e 11,91 cm, respectivamente. Desempenho semelhante foi verificado em Belo Oriente, Guanhães e Ipaba de 11,36; 12,52; e 11,79 cm, respectivamente. A altura apresentou as médias em Belo Oriente, Guanhães e Ipaba de 15,98; 18,23; e 17,46 m, respectivamente. Rocha, M.G.B. et al. (2007) observaram valores médios de 16,96 e 18,09 m para E. urophylla e E. grandis, respectivamente. Para o volume $\mathrm{em}^{3}$, as médias observadas em Belo Oriente, Guanhães e Ipaba de 0,07966; 0,10553; e 0,09209 m³ são comparáveis com os valores médios de 0,0943 e 0,1008 $\mathrm{m}^{3}$ em E. urophylla e E. grandis, respectivamente, observados por Rocha M.G.B. et al. (2007).

R. Árvore, Viçosa-MG, v.33, n.2, p.255-263, 2009 
Tabela 3 - Resultados da análise de variância das características DAP, ALT e VOL avaliadas nas progênies de meios-irmãos de eucalipto conduzidas em Belo Oriente, Guanhães e Ipaba

Table 3 - Results from the analysis of variance of the characteristics DBH (Diameter at Breast Height), H (Plant Height) and VOL (Wood Volume) evaluated in half-sib Eucalyptus progenies planted in Belo Oriente, Guanhães and Ipaba

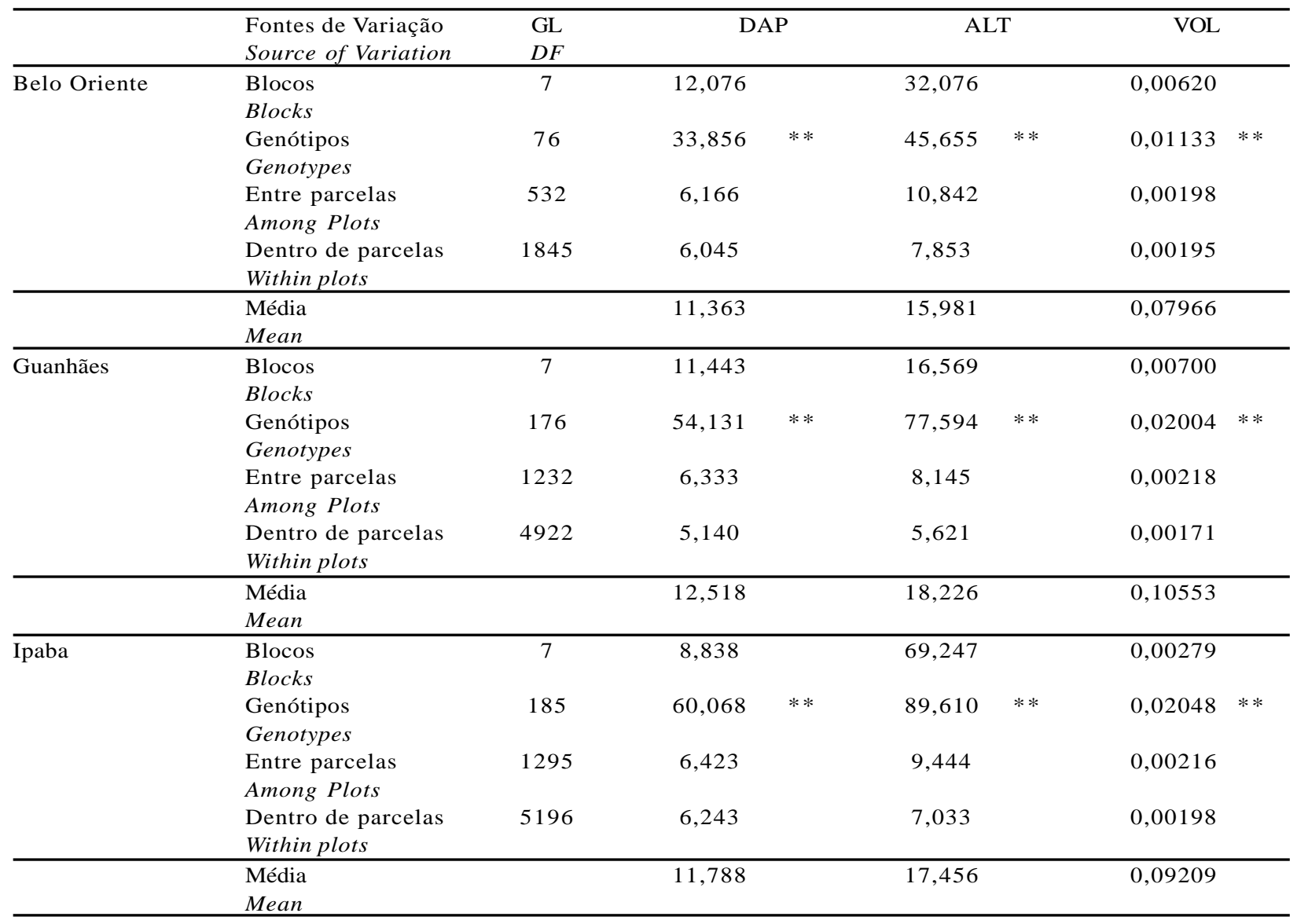

** - Diferença significativa a 1\% de probabilidade, pelo teste F, entre os genótipos; DAP - Diâmetro à Altura do Peito (cm); ALT - Altura de Planta (m); e VOL - Volume de Madeira (m3).

** - Significant difference at $1 \%$ probability by the F test among the genotypes; DAP - Diameter at Breast Height (cm); ALT - Plant Height $(m)$ and VOL - Wood Volume $(\mathrm{m} 3)$.

Teoricamente, a variância genética dentro de progênies de meios-irmãos é três vezes superior à variância entre as progênies, proporção essa observada na prática neste estudo (CRUZ, 2005). Entre os valores estimados do coeficiente de herdabilidade, a estimativa tendo como unidade de seleção a média entre progênies foi a que apresentou maior magnitude, atingindo valores próximos a $90 \%$ em Guanhães e Ipaba, quanto as características analisadas. Esses resultados indicam que o valor fenotípico das progênies são bons preditores dos valores genotípicos e que a seleção fenotípica entre progênies é eficiente.

R. Árvore, Viçosa-MG, v.33, n.2, p.255-263, 2009
Os coeficientes de variação experimentais obtidos das características DAP e ALT foram considerados de baixa magnitude, apresentando valores menores que $12 \%$, próximos aos obtidos por Muro-Abad et al. (2003). Nesse trabalho, os autores verificaram valores de altas magnitudes do CV quanto à característica VOL, valores esses que chegaram a 30\%, como em Belo Oriente.

Com a seleção massal, os ganhos estimados da característica DAP foram relativamente maiores em Guanhães e Ipaba (3,64 e 4,01 cm). O progresso genético predito com as diferentes estratégias de seleção apresentaram magnitudes de ganho semelhantes, 
indicando eficiência análoga das metodologias para a identificação dos melhores genótipos de adaptação específica aos ambientes (Tabela 5). Dessa forma, a eficiência de selecionar os indivíduos de melhor fenótipo no experimento, ou entre e dentro de famílias, considerando o valor genético aditivo de indivíduos e famílias, apresentou estimativas de progresso genético de magnitude semelhante.

A seleção massal, realizada para a característica ALT, propiciou menores estimativas de ganhos genéticos em Belo Oriente e Guanhães, comparativamente à Ipaba. Verificou-se que a seleção massal, massal estratificada, seleção entre e dentro de famílias e a seleção combinada apresentaram estimativas dos ganhos de seleção semelhantes, obtendo-se no máximo $24,06 \%$ de ganho, estimativa essa obtida da seleção combinada em Ipaba. Muro-Abad (2003) verificou ganhos para a seleção entre e dentro de famílias de meios-irmãos de Eucalyptus pellita, aos 3 anos de idade, que variaram de 1,77 a 12,68\% com relação à variável ALT.

A seleção da característica VOL propiciou maiores estimativas de ganhos em Ipaba. Assim como nas demais características, para essa também a seleção massal, massal estratificada, seleção entre e dentro de famílias e seleção combinada apresentaram estimativas de ganhos genéticos com magnitude semelhante $(102,18$; 101,$80 ; 74,76$; e 100,03\%, em relação aos valores observados em Ipaba). Tais resultados indicam que a seleção dos indivíduos de melhores fenótipos dentro do experimento, ou a prática da seleção com a mesma intensidade, dentro de bloco, ou combinada entre e dentro de famílias, obter-se-ão as estimativas de progresso genético semelhantes nas três características avaliadas.

Tabela 4 - Estimativa das variâncias entre blocos $\left(\sigma_{b}^{2}\right)$, genética entre progênies $\left(\sigma_{g}^{2}\right)$, genética dentro de progênies $\left(\sigma_{g \mathrm{~d}}^{2}\right)$, fenotípica dentro de progênies $\left(\sigma_{f d}^{2}\right)$, residual $\left(\sigma_{r}^{2}\right)$ e total $\left(\sigma_{t o t}^{2}\right)$; estimativa da herdabilidade, sendo a unidade de seleção média entre $\left(\sigma_{m f}^{2}\right)$, dentro de $\left(\sigma_{d f}^{2}\right)$, indivíduo no bloco $\left(\sigma_{I b}^{2}\right)$, indivíduo no experimento $\left(\sigma_{\text {Iexp }}^{2}\right)$; coeficiente experimental $(\mathrm{CV})$; coeficiente de variação genético entre $\left(\mathrm{CV}_{\mathrm{GE}}\right)$; e coeficiente de variação genético dentro $\left(\mathrm{CV}_{\mathrm{GD}}\right)$

Table 4 - Estimate of the variances among blocks $\left(\sigma_{b}^{2}\right)$, genetic variances among progenies $\left(\sigma_{g}^{2}\right)$, genetic variances within progenies $\left(\sigma_{g \mathrm{~d}}^{2}\right)$, phenotypic variances within progenies $\left(\sigma_{f d}^{2}\right)$, residual variance $\left(\sigma_{r}^{2}\right)$, total variance $\left(\sigma_{t o t}^{2}\right)$; estimate of heritability as the unit of average selection among progenies $\left(\sigma_{m f}^{2}\right)$, within progenies $\left(\sigma_{d f}^{2}\right)$, individual in the block $\left(\sigma_{I}^{2}\right)$, individual in the experiment $\left(\sigma_{\text {Iexp }}^{2}\right)$; experimental coefficient $(C V e)$, genetic variation coefficient among progenies $\left(C V_{G E}\right)$ and genetic variation coefficient within progenies $\left(C V_{G D}\right)$

\begin{tabular}{|c|c|c|c|c|c|c|c|c|c|}
\hline \multirow{2}{*}{$\begin{array}{l}\text { Parâmetros } \\
\text { Parameters }\end{array}$} & \multicolumn{3}{|c|}{ Belo Oriente } & \multicolumn{3}{|c|}{ Guanhães } & \multicolumn{3}{|c|}{ Ipaba } \\
\hline & DAP & ALT & VOL & DAP & ALT & VOL & DAP & ALT & VOL \\
\hline$\sigma_{b}^{2}$ & 0,0208 & 0,0803 & 0,000016 & 0,0068 & 0,0114 & 0,000007 & 0,0030 & 0,0756 & 0,000001 \\
\hline$\sigma_{g}^{2}$ & 0,9372 & 1,2674 & 0,000341 & 1,4014 & 2,0784 & 0,000534 & 1,5635 & 2,3567 & 0,000538 \\
\hline$\sigma_{g \mathrm{~d}}^{2}$ & 2,8117 & 3,8021 & 0,001022 & 4,2041 & 6,2353 & 0,001603 & 4,6904 & 7,0701 & 0,001615 \\
\hline$\sigma_{f d}^{2}$ & 6,0449 & 7,8534 & 0,001949 & 5,1401 & 5,6207 & 0,001712 & 6,2433 & 7,0331 & 0,001976 \\
\hline$\sigma_{r}^{2}$ & 0,0328 & 0,8703 & 0,000008 & 0,2799 & 0,6043 & 0,000113 & 0,0418 & 0,5670 & 0,000044 \\
\hline$\sigma_{t o t}^{2}$ & 7,0357 & 10,0714 & 0,002314 & 6,8281 & 8,314798 & 0,002365 & 7,8516 & 10,0324 & 0,002559 \\
\hline$\sigma_{m f}^{2}$ & 0,8179 & 0,7625 & 0,8256 & 0,883 & 0,895 & 0,8911 & 0,8931 & 0,8946 & 0,8944 \\
\hline$\sigma_{d f}^{2}$ & 0,4651 & 0,4841 & 0,5242 & 0,8179 & 1,1094 & 0,9365 & 0,7513 & 1,0053 & 0,8176 \\
\hline$\sigma_{I b}^{2}$ & 0,5344 & 0,5074 & 0,5929 & 0,8217 & 1,0012 & 0,9061 & 0,7968 & 0,9468 & 0,8419 \\
\hline$\sigma_{\text {Iexp }}^{2}$ & 0,5328 & 0,5034 & 0,5888 & 0,8209 & 9999 & 0,9036 & 0,7965 & 0,9396 & 0,8417 \\
\hline $\mathrm{CV}$ & 11,3711 & 11,1186 & 30,1119 & 9,7364 & 7,6619 & 21,6635 & 10,3812 & 8,5377 & 24,4938 \\
\hline $\mathrm{CV}_{\mathrm{GE}}$ & 8,5196 & 7,0442 & 23,1658 & 9,4567 & 7,9102 & 21,9047 & 10,6076 & 8,7945 & 25,1989 \\
\hline $\mathrm{CV}_{\mathrm{GD}}$ & 14,7564 & 12,201 & 40,1244 & 16,3795 & 13,7008 & 37,94 & 18,3728 & 15,2325 & 43,6458 \\
\hline $\mathrm{CV}_{\mathrm{GE}} / \mathrm{CV}$ & 5,3472 & 1,2068 & 6,6353 & 2,2377 & 1,8546 & 2,1762 & 6,1158 & 2,0387 & 3,4942 \\
\hline$\overline{\mathrm{CV}_{\mathrm{GD}} / \mathrm{CV}}$ & 9,2616 & 2,0902 & 11,4927 & 3,8758 & 3,2122 & 3,7693 & 10,5929 & 3,5311 & 6,0522 \\
\hline
\end{tabular}

DAP - Diâmetro à Altura do Peito $(\mathrm{cm})$, ALT - Altura de Planta $(\mathrm{m})$ e VOL - Volume de Madeira (m3).

DAP - Diameter at Breast Height (cm); ALT - Plant Height $(\mathrm{m})$ and VOL - Wood Volume $(\mathrm{m} 3)$. 
Tabela 5 - Ganhos genéticos obtidos com seleção massal, seleção massal estratificada, seleção entre e dentro de progênies e seleção combinada, em Belo Oriente, Guanhães e Ipaba

Table 5 - Genetic gains obtained by mass selection, stratified mass selection, selection among and within progenies and combined selection in Belo Oriente, Guanhães and Ipaba

\begin{tabular}{|c|c|c|c|c|c|c|c|c|c|}
\hline \multirow{2}{*}{$\begin{array}{l}\text { Método de Seleção } \\
\text { Method of Selection }\end{array}$} & \multicolumn{3}{|c|}{ Belo Oriente } & \multicolumn{3}{|c|}{ Guanhães } & \multicolumn{3}{|c|}{ Ipaba } \\
\hline & DAP & ALT & VOL & DAP & ALT & VOL & DAP & ALT & VOL \\
\hline$\overline{\text { Massal }}$ & $2,7795 *$ & 2,7879 & 0,0661 & 3,6402 & 3,3595 & 0,0883 & 4,0112 & 4,5175 & $\overline{0,0941}$ \\
\hline Massal & $(24,46) * *$ & $(17,44)$ & $(82,99)$ & $(29,08)$ & $(18,43)$ & $(83,69)$ & $(34,03)$ & $(25,88)$ & $(102,18)$ \\
\hline Massal estratificada & 2,7624 & 2,7587 & 0,0657 & 3,6266 & 3,2721 & 0,0878 & 4,0086 & 4,4545 & 0,0938 \\
\hline Stratified massal & $(24,31)$ & $(17,26)$ & $(82,48)$ & $(28,97)$ & $(17,95)$ & $(83,17)$ & $(34,01)$ & $(25,52)$ & $(101,80)$ \\
\hline $\begin{array}{l}\text { Entre e dentro } \\
\text { de progênies }\end{array}$ & 2,4328 & 2,3338 & 0,0533 & 2,8090 & 2,4113 & 0,0656 & 3,1244 & 3,4093 & 0,0689 \\
\hline $\begin{array}{l}\text { Among and within } \\
\text { progenies }\end{array}$ & $(21,41)$ & $(14,60)$ & $(66,87)$ & $(22,44)$ & $(13,23)$ & $(62,15)$ & $(26,51)$ & $(19,53)$ & $(74,76)$ \\
\hline Seleção combinada & 2,9969 & 2,9945 & 0,0668 & 3,6444 & 3,2750 & 0,0853 & 4,0125 & 4,4447 & 0,0921 \\
\hline Combined selection & $(26,37)$ & $(18,74)$ & $(83,86)$ & $(29,11)$ & $(17,97)$ & $(80,90)$ & $(34,04)$ & $(25,46)$ & $(100,03)$ \\
\hline
\end{tabular}

*- Ganhos genéticos; **- Ganhos genéticos em porcentagem; DAP - Diâmetro à Altura do Peito (cm); ALT - Altura de Planta (m); e VOL

- Volume de Madeira (m3).

*-Genetic gains; ** - Genetic gains in percentage; DAP - Diameter at Breast Height (cm); ALT - Plant Height (m); and VOL - Wood Volume (m3).

Para a característica VOL, Muro-Abad (2003) obteve ganhos que variaram de 8,15 a 34,81\%, dependendo do local de avaliação com seleção entre e dentro de progênies de meios-irmãos. Esses ganhos foram inferiores aos estimados neste experimento, quando se empregou a mesma estratégia de seleção, o que se deveu a variabilidade e à eficiente predição dos valores genéticos a partir dos valores fenotípicos, subsidiados pelos valores de herdabilidades de alta magnitude observados nas características.

Dessa forma, considerando questões práticas, entende-se que para essa população o método de seleção massal propicia maior custo/benefício, devido à sua simplicidade e eficiência semelhante.

\section{CONCLUSÕES}

A população estudada apresentou bom potencial e estimativas de progresso genético com a prática da seleção.

A seleção massal, massal estratificada, seleção entre e dentro de famílias e a seleção combinada apresentaram estimativas semelhantes dos ganhos genéticos, devido aos valores elevados com relação às estimativas de variabilidade genética e herdabilidades.

As maiores estimativas dos ganhos com a seleção foram obtidas em Ipaba.

R. Árvore, Viçosa-MG, v.33, n.2, p.255-263, 2009

\section{REFERÊNCIAS}

Anuário Estatístico da ABRAF. Ano Base 2007/ Brasília: 2008. 90p.

ALLARD, R.W. Principles of plant breeding. New York, John Wiley \& Sons, 1960. 485p.

CRUZ, C. D. Programa GENES - aplicativo computacional em genética e estatística. Viçosa, MG: Universidade Federal de Viçosa, 2001. 542p.

CRUZ, C. D. Princípios de genética quantitativa; Viçosa, MG: Universidade Federal de Viçosa, 2005. 394p.

CRUZ, C. D.; REGAZZI, A. J.; CARNEIRO, P. C. S. Modelos biométricos aplicadas ao melhoramento genético. 3.ed. Viçosa, MG: Universidade Federal de Viçosa, 2004. v.1. 480p.

EMPRESA BRASILEIRA DE PESQUISA AGROPECUÁRIA - EMBRAPA.

Importância socioeconômica e ambiental no cultivo do Eucalipto. 2006. Disponível em: <http:// sistemasdeproducao.cnptia.embrapa.br/ FontesHTML/Eucalipto/CultivodoEucalipto/ 01 01 historico.htm $>$ Acessado em 09 de maio de 2006. 
FAO, 2003 - Forest products Disponível em: <http://apps1.fao.org/servletteServlet.jrum?Areas+

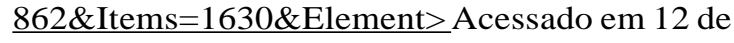
abr. de 2003.

FREITAS, M. L. M. et al. Variação genética em progênies de Myracrodruon urundeuva F.F. \& M.F. Allemão em três sistemas de cultivo.

Revista Árvore, v.30, n.3, p.319-329, 2006.

GARDNER, O. G. Estimates of genetic parameters in cross fertilizing plants and their implications in plant breeding. In: HANSON, W. D.; ROBINSON, H. F. (Eds). Statistical genetics and plant breeding. New York: National Academy of Science, 1963. p.225-252.

MARTINS, I. S.; CRISTO, R. C.; CORREIA, H. S. Comparação entre seleção combinada e seleção direta em Eucalyptus grandis, sob diferentes intensidades de seleção. Floresta e Ambiente, v.8, n.1, p.36-43, 2001.

MARTINS, I. S. et al. Eficiência da seleção univariada direta e indireta e de índices de seleção em Eucalyptus grandis, Revista Árvore, v.27, n.3, p.327-333, 2003.

MARTINS, I. S. et al. Comparação entre os processos de seleção entre e dentro e o de seleção combinada, em progênies de Eucalyptus grandis, Cerne, v.11, n.1, p.16-24, 2005.

MORAES, M. L. T. Variação genética da densidade básica da madeira em progênies de Eucalyptus grandis Hill ex Maiden e suas interações em as características de crescimento. 1987. $115 \mathrm{f}$. Dissertação (Mestrado em Ciência Florestal) Escola Superior de Agricultura Luiz de Queiroz, Piracicaba, 1987.
MURO-ABAD，J. I. Diversidade genética por marcadores moleculares e predição de ganhos em Eucalyptus spp. 2003. 98f. Tese (Doutorado em Genética e Melhoramento) - Universidade Federal de Viçosa, Viçosa, MG, 2003.

PAULA, R. C. et al. Predição de ganhos genéticos em melhoramento florestal. Pesq. Agropec. Bras., v.37, n.2, p.159-165, 2002.

PIRES, I. E. Eficiência da seleção combinada no melhoramento genético do Eucalyptus spp. 1996. 116f. Tese (Doutorado em Genética e Melhoramento) - Universidade Federal de Viçosa, Viçosa, MG, 1996.

REIS, G. G. et al. Crescimento de raizes e da parte aérea de clones de híbridos de Eucalyptus grandis x Eucalyptus urophylla e de Eucalyptus camaldulensis $\mathrm{x}$ Eucalyptus spp submetidos a dois regimes de irrigação no campo. Revista Árvore, v.30, n.6, p.921-931, 2006.

ROCHA, M. G. B. et al. Seleção de genitores de Eucalyptus grandis e de Eucalyptus urophylla para produção de híbridos interespecíficos utilizando REML/BLUP e informação de divergência genética. Revista Árvore, v.31, n.6, p.977-987, 2007.

TORREGOZA, M.; HARDSTEAD, D. D. Effects of mass selection for ears per plant is maize. In: Agronomy abstracts. Madison, ASA, 1967. p.20.

VENCOVSKY, R. Genética quantitativa. In: KERR, W.E. (Org). Melhoramento e genética. São Paulo: Melhoramento, 1969. p.17-38. 